\title{
Autonomic Response Specificity during Motor Imagery
}

\author{
Kazuo Oishi' ${ }^{1)}$, Tatsuya Kasai ${ }^{2)}$ and Takashi Maeshima ${ }^{1)}$ \\ 1) Health and Sports Sciences Institute, Senshu University \\ 2) Division of Sports and Health Sciences, Graduate School for International \\ Development and Cooperation, Hiroshima University
}

\begin{abstract}
It has been reported that activation of autonomic effectors during mental simulation of voluntary motor actions (motor imagery: MI) may be explained by two different factors, i.e., functions of preparation or anticipation of actual exercise (motor anticipation) and the central motor programming/ planning which acts during actual motor action (motor programming). This study was designed to clarify how these factors participate during MI, utilizing two mental tasks with high mental stress, i.e., MI and mental arithmetic (MA). Several autonomic effectors' responses were compared between MI of a $500 \mathrm{~m}$ speed skating sprint and MA. Subjects were eight 18 to 25 year old young male speed skate athletes, all of them could easily and vividly imagine a $500 \mathrm{~m}$ speed skating sprint. Duration of the MI ranged from 35 to $38 \mathrm{sec}$ and these were very close to each subject's actual best record (means of absolute differences were less than $0.6 \mathrm{sec}$, i.e., less than $1.7 \%$ relatively). A significant decrease of skin resistance (SR), increases of heart rate (HR) and respiration rate were observed in both MI and MA when compared to each control resting level (excluding one subject for respiration rate during MI). SR decreased during MI (mean and SD of 8 subjects: $45.9 \pm 17.7 \%$ ) and MA $(39.7 \pm 16.8 \%)$, with no significant differences between MI and MA ( $\mathrm{t}=1.29$, by paired t-test). HR increased significantly above control values in MA (10.3 \pm $4.3 \%)$ and MI (44.3 $\pm 18.8 \%)$. However, the increase during MA was significantly smaller $(t=4.99, \mathrm{p}<0.001)$ than in MI. Respiratory rate increased significantly in both MI $(46.5 \pm 30.9 \%)$ and MA $(27.7 \pm 14.6 \%)$, with no significant difference between MI and MA $(\mathrm{t}=1.82)$ due to the large individual variation in MI. The frequency of respiration was fairly regular during MA, but quite irregular during MI (similar to those during actual motor actions). The central nervous system which acts in MI may possess the function of activation of target effectors which play an important role in actual exercise, on the basis of incremental vigilance level induced by the function of motor anticipation. J Physiol Anthropol 19 (6): 255-261, 2000 http://www.jstage.jst.go.jp/en/
\end{abstract}

Keywords: motor imagery, skin electroresistance, heart rate, respiration activity, motor programming, motor anticipation

\section{Introduction}

Mental activity and stress induce many autonomic effectors' responses. These responses are typically an elevation of heart rate, blood pressure, respiration rate, and electrodermal activity. It is well known that these autonomic responses are observed during preparation or anticipation of actual exercise (motor anticipation) (Lang et al., 1978). These responses are considered as components of behavioral responses in the central nervous system (Collet et al., 1994) and may reflect cognitive processes (Deschaumes-Molinaro et al., 1991; Lacey and Lacey, 1970; Wallin and Fagius, 1986). During mental simulation of voluntary motor actions (motor imagery: MI), similar responses of autonomic effectors are also observed without any actual muscle movement (Decety et al., 1991; Drummond et al., 1978). These responses have been considered functionally similar with motor anticipation based on the elevation of vigilance level (Collet et al., 1994; Decety et al., 1993).

Other interpretations exist for the autonomic response observed during MI. Studies of regional cerebral blood flow indicate that specific cerebral regions such as the cerebellum, the basal ganglia, and the supplementary motor area are all activated not only during actual movements but also during MI (Decety et al., 1990; Ingver and Philipson, 1977; Oishi et al., 1992; Pauli et al., 1994, Roland et al., 1980, Ryding et al., 1993). From time estimation studies during MI, it has been reported that the duration of the actual exercise (such as walking, running, or skating) is similar to those during MI (Decety et al., 1989; Oishi et al., 1994; Yasukawa et al., 1993). The magnitude of activation in each autonomic effector has been shown to be proportional to the degree of mental effort in MI such that more difficult MI task elicits a greater autonomic response (Decety et al., 1991, 1993). A 
more direct finding has been reported by Yue and Cole (1993) who have shown that a MI training program of a repetitive digit muscle contraction task can produce improvements in the force and endurance capacity of the muscle. It has been suggested, based on all of the above findings that the central motor programming/planning which acts during actual motor action (motor programming) also participates in autonomic responses during MI. This is based on the idea that the motor programming involves not only the motor but also the autonomic function. However, in most cases only the autonomic effectors' activities have been observed, since the final output of the motor function may be inhibited during MI (Decety et al., 1993; Oishi et al., 1994).

Two factors can account for autonomic activation during MI: 1) the function of motor anticipation as the components of behavioral responses in central nervous system, and 2) the function of motor programming which may be set in higher brain centers. However, there has been much confusion regarding these factors, and it is not clear how these factors participate during MI.

This study was designed to clarify, in part, roles of motor anticipation and motor programming on autonomic responses utilizing two mental tasks, i.e., MI and mental arithmetic (MA). Participated athletes had been trained for MI and have excellent MI performance. These athletes produce a greater autonomic response to imagined exercise due to their prior physical and mental training (Wuyam et al., 1995). These subjects were asked to perform MI with maximum effort (such as that induced by the Olympic games), and also were instructed to conduct MA as precisely and as correctly as possible. Autonomic responses were then compared between these two mental tasks.

\section{Method}

\section{Subjects}

Eight Japanese male speed skate sprinters volunteered. The group consisted of national team members including three Olympic game athletes who were 18 to 25 years old. Each subject received a complete explanation of the experimental test procedure prior to their consent.

Subjects had all been utilizing MI for 5 to 60 months previously in their study due to the fact that MI is a part of the image training program for speed skate competitors in Japan. All subjects could imagine the vivid imagery of skating without difficulty.

\section{Recordings of autonomic variables}

Electrodermal activity, heart rate (HR), and respiratory activity were recorded simultaneously on an analog magnetic tape recorder, and analyzed separately. HR was recorded by standard electrode placement in the V5 position. Electrodermal activity was expressed by skin resistance (SR) utilizing two $\mathrm{Ag}-\mathrm{AgCl}$ electrodes set on the fore and middle fingers. Respiration curve was recorded as resistive changes from a thermistor mounted across the nostril on the basis of the temperature difference between inhaled and exhaled air. Respiration rate and $\mathrm{HR}$ were analyzed by a computer analysis system (NEC Medical System Inc.). The SR signals from the tape recorder were digitized at $10 \mathrm{~Hz}$ sampling frequency by a computerized SR analysis system (NEC Medical System Inc.). SR signals were recorded for qualitative obsevation, and then average SR levels during $40 \mathrm{sec}$ were compared between resting and mental tasks (MI or MA) for quantitative assessment (see Data analysis). All of these procedures were similar to former study (Oishi et al., 1994).

\section{Mental tasks}

Motor imagery (MI): The subjects lay supine, and awake but relaxed, on a comfortable bed. To provide a consistent environment for the best possible concentration on MI for each subject, the following procedures were performed:

A rest period of 5 minutes followed preparation of the electrical recordings. All recordings of physiological variables were initiated at this time. After the initial period, subjects were instructed to relax for 4 minutes using an original positive relaxation technique (Oishi et al., 1992), and to mentally concentrate for an additional 2.5 minutes for MI. Immediately after this concentration period, an announcement for the start of the $500 \mathrm{~m}$ sprint was very similar to those used in actual athletic meets. Then MI was started by the sound of a signal gun and continued to the end of the simulated $500 \mathrm{~m}$ speed skate sprint. To avoid occurrence of a startle response, the volume of the starter gun was carefully controlled.

Experimental procedures were given from tape recorded instructions for consistency between experiments. Each subject repeated the experiment 4 times on different days. The duration of each simulated race was timed by the subject, who was instructed to start a stop watch at the beginning of the MI and stop at the finish line. Previous data from the authors indicate no obvious effect on autonomic data by the movement required for stop watch operation (Oishi et al., 1994). Each MI was continued for about $35 \mathrm{sec}$ to $38 \mathrm{sec}$ judging from the stop watch, and each duration was very close to the subject's personal actual best records (mean of absolute differences was less than $0.6 \mathrm{sec}$, i.e., less than $1.7 \%$ as the relative difference).

Mental arithmetic (MA): The subjects lay supine on the bed as in the MI trial. Subjects were instructed to perform 40 seconds of continuous calculations mentally (mental arithmetic: MA). The subjects subtracted 7 in succession from triple figures given by the investigator (e.g., 223). Since task difficulty may relate to induced physiological 
responses (Callister et al., 1992; Pauli et al., 1994), MA was instructed to be conducted as fast and as precisely as possible without any motor involvement such as finger movement.

Following the preparation for the electrical recordings, the subjects rested supine for 5 minutes. After this initial rest period, the subjects were instructed to relax for 4 minutes using the original positive relaxation technique as in the MI trial. Subjects were then instructed to initiate MA at the investigator's cue. Each subject repeated this experiment 4 times on different days.

\section{Structured interview}

Each subject was instructed to have a structured interview. One of them was applied before initiation of this study, including several questions about actual skating, e.g., how do you breath in actual skating? What is your athletic ranking and best record?, etc. The other interviews were applied just after each experimental trial. These consisted of several questions about MI and MA including the following ratings.

After each MI trial, the subjects were instructed to rate on a vividness scale consisting of five degrees from "completely no appearance" (rating 1) to "fully vivid, just like the actual experience" (rating 5). This rating scale was based on the authors' previous study (Yasukawa et al., 1993). Additionally, the subjects were also instructed to rate on a perspective rating scale consisting of five degrees from "completely external, much like in home movies" (rating 1) to "completely internal, just like the actual experience" (rating 5). This rating scale was developed by authors based on the report of Mahoney and Avener (1977).

Also after each MA trial, a perceived stress scale was presented to subjects which consisted of five degrees from "not stressful" (rating 1) to "very very stressful" (rating 5). Subjects reported their numerical ratings of stress and the rating was recorded. This rating scale was developed by Callister et al. (1992).

\section{Data analysis}

The MA and MI trials for each subject were conducted on different days, and randomly ordered. Only the MI experiment in which each subject judged to be the most vivid and completely internal was selected for analysis. Similarly, the MA in which each subject judged to be the most stressful was selected (see discussion).

Baseline data from the autonomic parameters were taken from the first 40 seconds of the initial rest period. SR data were sampled every second throughout each test. Respiratory rate and $\mathrm{HR}$ were calculated as instantaneous values each minute, using each peak to peak interval. Changes in all variables were calculated as the relative values based on the baseline data. Statistical evaluation of mean differences were analyzed using the
Student's t test.

\section{Results}

All of the measurements for SR, HR, and respiratory rate changed significantly during both MI and MA compared with each control base level for each subject (excluding respiratory rate in MI trial of one subject). However, the magnitude of the change and/or the change manner of time course in each variable were different between MI and MA.

\section{Skin resistance (SR)}

After the initiations by cues, continuous decreases were observed in both MI and MA. The range of the average decreases of SR level during $40 \mathrm{sec}$ in 8 subjects was from $27 \%$ to $78 \%$ (absolute differences; from 25 to $273 \mathrm{k} \Omega$ ) in MI and from $10 \%$ to $133 \%$ (from 21 to $249 \mathrm{k} \Omega$ ) in MA respectively (Fig. 1). The magnitude of these decreases was large in both MI (mean \pm SD of 8 subjects: $45.9 \pm$ $17.7 \%)$ and MA (39.7 $\pm 16.8 \%)$. There was no statistical significant difference between these two means by paired $\mathrm{t}$-test $(\mathrm{t}=1.29)$.

\section{Heart rate (HR)}

HR increased after the task initiation and continued during the tasks. The range of the increases in 8 subjects was from $24 \%$ to $77 \%$ (from 13.9 to 53.9 beats/min) in MI and from $4 \%$ to $17 \%$ (from 2.5 to 10.2 beats $/ \mathrm{min}$ ) in MA. The magnitude of the HR increase was significantly higher in MI than that in MA $(44.3 \pm 18.8 \%$ in MI, $10.3 \pm$ $4.3 \%$ in $\mathrm{MA}, \mathrm{t}=4.99, \mathrm{p}<0.001$, by paired t-test). HR showed the largest difference in percentage between MI and MA tasks compared with the other autonomic variables (Fig. 2).

\section{Respiration}

Respiratory rates increased after the initiation of MA and MI tasks. However, there was one subject whose respiratory rate was slightly decreased $(-6 \%)$ during MI, even though a significant increase (11\%) was observed during MA in this subject.

The time course of the respiratory curve was extremely different between the two mental tasks (Fig. 3). Respiration during MI was activated quite irregularly. Additionally, after the announcement of the start, a short pause of respiration just before the sound of the signal gun, just like an actual situation, could be observed in all of the MI tasks. In contrast, respiration was activated quite regularly during MA.

The range of the increases in 8 subjects was from - $6 \%$ to $100 \%$ (from -1.1 to $18.0 \mathrm{cycles} / \mathrm{min}$ ) in $\mathrm{MI}$ and from $5 \%$ to $47 \%$ (from 0.9 to 8.5 cycles/min) in MA. Means and standard deviations of the increment of respiratory rate in 8 subjects were $46.5 \pm 30.9 \%$ in MI and $27.7 \pm 14.6 \%$ in 


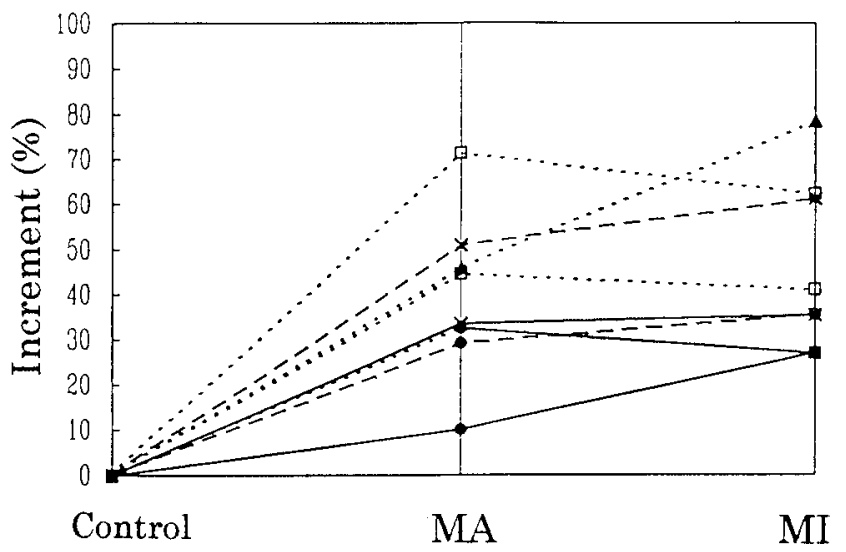

Fig. 1 Increment of the level of skin resistance (SR) during mental arithmetic (MA) and motor imagery (MI) for 8 subjects. Different lines and symbols show different subjects. Each measurement was indicated by relative values compared with that of control base level. Means of the decreases in 8 subjects were $39.7 \pm 16.8 \%$ and $45.9 \pm 17.7 \%$ for MA and MI respectively. The difference between those means was not significant statistically by paired $\mathrm{t}$-test $(\mathrm{t}=1.29)$.

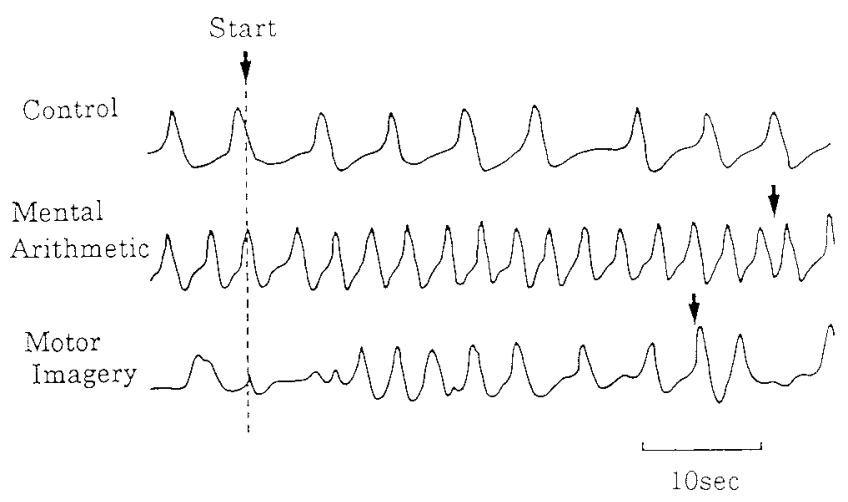

Fig. 3 Samples of respiratory curves from one subject during two different mental tasks and control rest period. Two arrows at the right show the end of each task. The variation in the cycle was larger during MI than that during MA. Control: $10.7 \pm 1.5$ cycle/ min (Coefficient of variance (CV): 13.6\%), MA: $21.9 \pm 2.5$ cycle/ min (CV: $11.3 \%$ ). MI: $21.1 \pm 7.5$ cycle/min (CV: $35.4 \%$ ).

MA respectively (Fig. 4). Since individual variation in the increase of the rate was large during MI, the difference of these increases during MI and MA trials was not significant ( $\mathrm{t}=1.82)$.

\section{Relationships in magnitudes of responses between $M I$ and $M A$}

Relationships of relative increments between MA and MI in three different autonomic variables for 8 subjects are shown in Figure 5. In SR, a significant correlation coefficient was obtained between MI and MA ( $r=0.726$, $\mathrm{p}<0.01)$. Namely, there was a statistically significant

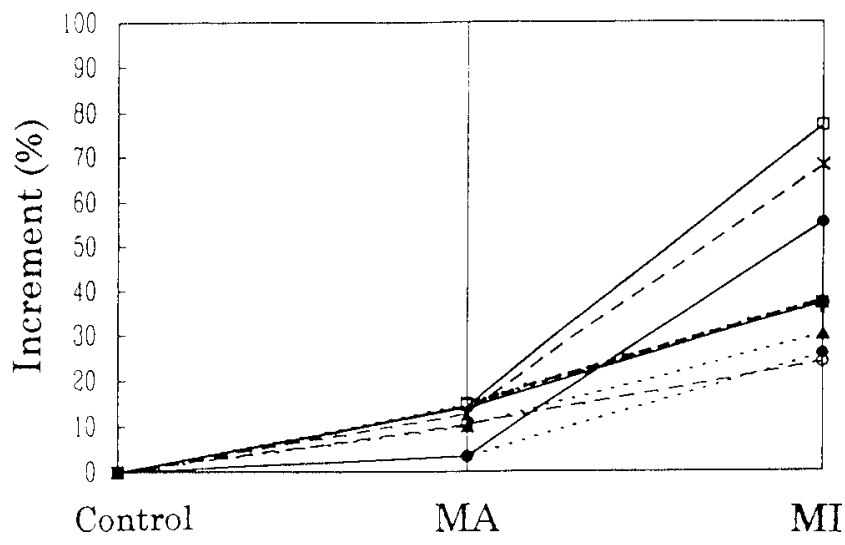

Fig. 2 Increment of heart rate during two mental tasks for 8 subjects. Different lines and symbols show different subjects. The means of the increments were $10.3 \pm 4.3 \%$ and $44.3 \pm 18.8 \%$ for MA and MI respectively. The mean of MI was significantly larger than that of MA $(\mathrm{t}=4.99, \mathrm{p}<0.001$, by paired $\mathrm{t}$-test $)$.

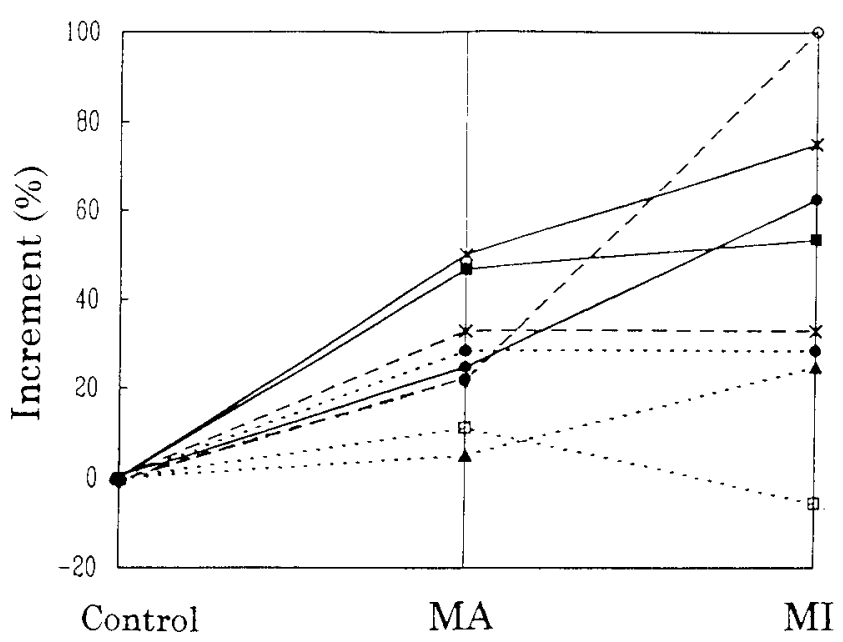

Fig. 4 Increments of respiratory rate during two mental tasks for 8 subjects. Different lines and symbols show different subjects. The means of the increments were $27.7 \pm 14.6 \%$ (MA) and 46.5 $\pm 30.9 \%$ (MI). The difference between those means was not significant statistically by paired $\mathrm{t}$-test $(\mathrm{t}=1.82)$.

tendency such that a subject who indicated greater response in SR during MI showed greater response during $\mathrm{MA}$, and vise versa. However, in $\mathrm{HR}(\mathrm{r}=0.288)$ and in respiratory rate $(\mathrm{r}=0.467)$, there were no statistically significant relations between MA and MI.

\section{Discussion}

\section{Evaluation of MI by structured interviews}

Sense of exertion during actual movement is produced by signals from several receptors in muscle and the surrounding tissues, and/or motor commands from 

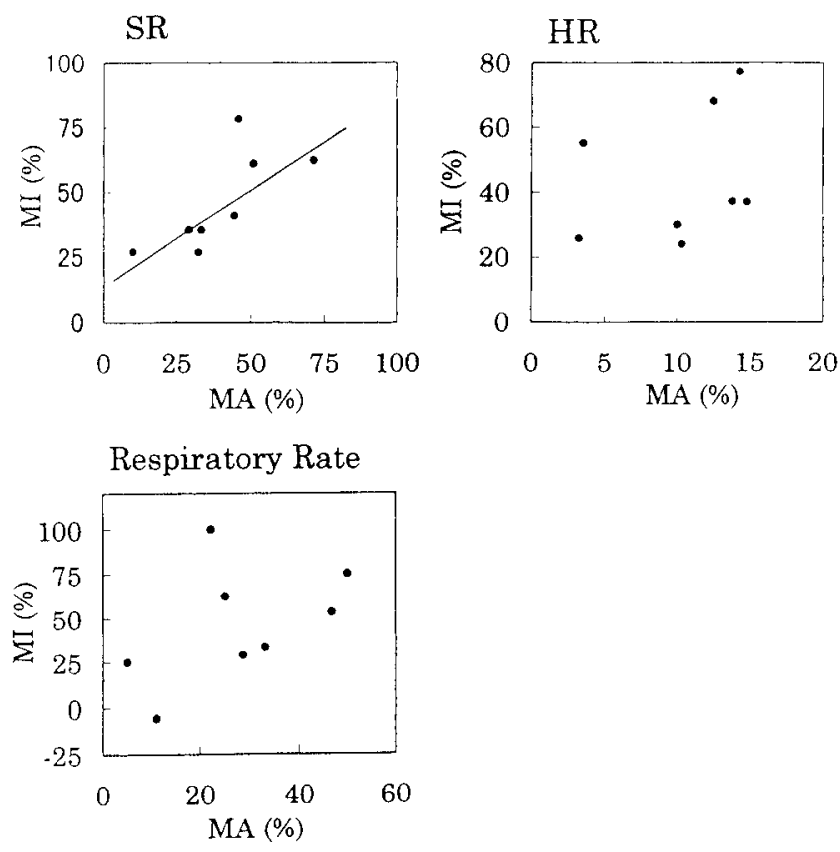

Fig. 5 Relationships of relative changes between MA and MI in three different autonomic variables for 8 subjects. In SR, significant relation was obtained between MI and MA ( $\mathrm{r}=0.726, \mathrm{p}<0.01)$. The slope and $y$-intercept of this regression line were 0.691 and 15.64 respectively. There were no significant relations in either $\mathrm{HR}(\mathrm{r}=0.288)$ and respiratory rate $(\mathrm{r}=0.467)$.

higher center (Aniss et al., 1988; Gandevia et al., 1983). It is noted that these sensations can be evaluated by the subjects even during MI in which the peripheral afferent feedback might be absent or extremely limited if it is existing (Decety et al., 1993). In this study, the subjects also reported in the structured interviews that several kinds of sense (sometimes included even a smell of surroundings) could be perceived "just like an actual skating", even though it was an imagined one.

Several perceived ratings which relate to MI may have some relations to subsequent physiological responses. The vivid and internal MI induces larger physiological responses than obscure and external MI (Hale, 1982; Wang and Mogan, 1992). Ratings of these variables were considered to have relations to motor programming activation (Oishi et al., 1994, Yasukawa et al., 1993). Therefore, only the MI experiment in which each subject judged to be the most vivid and completely internal was selected for analysis. Similarly, the MA in which each subject judged to be the most stressful was selected. Concequently, the MIs in which the vividness scale rating and the perspective rating indicated both 5 , and the MAs in which the stress scale rating indicated 5 were selected in all cases. This selection did not show a specific pattern in order of each task in both MA and MI, such that the most stressful MA trials were obtained in the first trial in all subjects. Additionally, Spearman's correlation coefficients (by ranks) in response to the magnitudes of physiological parameters among different trials did not show significant relations $(<0.3)$ in all cases. Therefore, the habituation in physiological responses was not observed both in MA and MI trials.

\section{Comparison of autonomic responses between MI and $M A$}

In former reports in regard to MI (Decety et al., 1991; Drummond et al., 1978; Oishi et al., 1992), the autonomic effectors' activities which represent sympathetic nervous system activity became greater in almost all the cases. In the present study, significant changes in HR, SR, and respiration rate were observed during MI compared with those during resting control in each subject (excluding one subject of respiratory rates in MI). Characteristic autonomic activation in each autonomic variable was observed during MI in comparison with MA data.

Additionally, considering the facts that, by structured interviews, each duration of the analyzed MI was very close to the subject's personal actual best records, vividness was excellent and perspective was completely internal, it is considered that the central motor programming was conducted during MI.

\section{$S R$}

Collet et al. (1994) have reported that electrodermal activity is well correlated to the evolution of vigilance (i.e., an increase in SR is related to subjects' relaxation level. Conversely, a decrease in SR would characterize an activation phase). SR is the most reliable index of activation level, and the tonic level of SR expresses the arousal evolution through the activation/relaxation axis (Collet et al., 1994).

SR decreased significantly during MI and MA in all subjects compared with the control resting levels. The magnitudes of these decreases were large in both MI and MA, and there were no obvious differences in magnitude and change time course between the two mental tasks. Therefore, it would be difficult to explain the interaction of the two functions between motor anticipation and motor programming from SR data.

From the correlation coefficient in SR decreases between MA and MI, there was a statistically significant tendency such that a subject who indicated greater response in SR during MI showed greater response during $\mathrm{MA}$, and vise versa. It is suggested that, in SR, individual difference in stress response was also reflected during MI. SR may be one of the variables which are difficult to show the effect from the function of the central motor programming.

$H R$
Callister et al. (1992) reported, using a 
microneurography technique, that the elevation in HR during MA may be only necessary for the adjustment to basic cognitive processes involved in MA, and clearly different from the other autonomic regulations. For example, sympathetic nerve activity to skeletal muscle (MSNA) has been shown to be proportional to the degree of stress, such that a higher stress elicits a larger autonomic response in MSNA. Conversely, only a slight increase in HR could be observed even if very stressful MA was applied. They concluded that the magnitude of the increment of HR was dependent upon the task difference and independent of stress magnitude.

In this study, HR was the typical variable in which the difference in the activation magnitude between MI and MA was greater than those for other variables. HR was increased significantly both in MI and MA, however the magnitude of the increment was extremely greater during MI compared with that during MA. These findings are considered to support the report by Callister et al. (1992). Additionally, the correlation coefficient in HR increases between MA and MI showed extremely low value. In other words, individual difference in HR response to stress for each subject was not reflected during MI. It is suggested that HR was one of the typical variables which well relate to the function of the central motor programming.

\section{Respiration}

Respiration is one of the commonest variables which have been used in MI studies (Decety et al., 1991, 1993; Deschaumes-Molinaro et al., 1992; Oishi et al., 1992, 1994; Wuyam et al., 1995). Usually respiration is activated not only in executions of MI but also in other types of stressful mental tasks. However, it is not yet understood whether respiration is activated differently or not between MI and the other mental tasks.

It is reported that respiration is activated regularly, during MI of regularly repeated motor actions (Decety et al., 1993; Wuyam et al., 1995). In contrast, in actual $500 \mathrm{~m}$ speed skating in which the movement is quite complicated, it was clear from the structured interviews that respiration was usually activated irregularly. There were large individual differences in the activation manner. During MI, respiration presented a very complicated curve (Fig. 4). There was one subject whose respiratory rate slightly decreased during MI. In the other subjects, respiration increased significantly more than 23\%. Based on the structured interviews with each subject, it was concluded that the pattern of respiration during MI was similar to that during actual skating, even in the subject whose respiratory rate decreased.

It was shown that the average frequencies of respiration during MI and MA were not different significantly, since individual variation in the increase of the rate was large during MI. However, the time course of the respiration curve was quite different between two mental tasks. Respiration was activated quite regularly during MA. From this finding, the rise of vigilance level without motor programming activation may increase the rate of respiration regularly. Conversely, it could be seen that the respiration during MI was activated quite irregularly and similar to that during actual skating sprints as mentioned above. Additionally, it was shown that the correlation coefficient for the increases of respiratory rate between MA and MI was not significant. The above findings suggested that the respiration control was modified largely by some factors during MI.

Tobin et al. (1986) showed that the increase in respiratory rate occurred remarkably during anticipation of actual exercise. Colebatch et al. (1991) noted that those similar specific cerebral regions which participated in actual motor actions, i.e., the cerebellum, the basal ganglia, and SMA, were activated during voluntary control of respiration. Considering these reports in addition to the findings obtained in this study, it is suggested that the activation of respiration is governed strongly by both factors, i.e., the functions of the motor anticipation and the central motor programming.

\section{Possible neural mechanisms for autonomic responses during $M I$}

Autonomic responses were usually observed during usual mental tasks, particularly including mental stress, without any MI at all. These responses are considered to be related to the function of motor anticipation or some behavioral functions in the autonomic nervous system. In contrast, the characteristic breathing or the greater increase of HR observed during MI could not be explained without the function of motor programming.

Deschaumes-Molinaro et al. (1992) and Collet et al. (1994) have presented the verified Lacey's hypothesis on individual autonomic response, i.e., each subject responds through a preferential autonomic nervous system channel. The central nervous system which acts in MI may possess a function of activation of target effectors which play an important role in actual exercise. Additionally, the action may conduct on the basis of incremental vigilance level induced by the function of motor anticipation.

\section{References}

Aniss AM, Gandevia SC, Milne RJ (1988) Changes in perceived heaviness and motor command produced by cutanous reflexes in man. J Physiol 397: 113-126

Callister R, Suwarno NO, Seals DR (1992) Sympathetic activity is influenced by task difficulty and stress perception during mental challenge in humans. J Physiol 454: 373-387

Colebatch JG, Adams L, Murphy K, Martin AJ, 
Lammertsma AA, Tochon-Danguy HJ, Clark JC, Friston KJ, Guz A (1991) Regional cerebral blood flow during volitional breathing in man. J Physiol 443: 91-103

Collet C, Deschaumes-Molinaro C, Delhomme G, Dittmar A, Vernet-Maury E (1994) Autonomic responses correlate to motor anticipation. Behav Brain Res 63: 71-79

Decety J, Jeannerod M, Germain M, Pastene J (1991) Vegetative response during imagined movement is proportional to mental effort. Behav Brain Res 42: 1-5

Decety J, Jeannerod M, Durozard D, Baverel G (1993) Central activation of autonomic effectors during mental simulation of motor actions in man. J Physiol 461: 549563

Decety J, Jeannerod M, Prablanc C (1989) The timing of mentally represented actions. Behav Brain Res 34: 3542

Decety J, Sjoholm H, Ryding E, Stenberg G, Ingvar DH (1990) The cerebellum participates in mental activity: tomographic measurements of regional cerebral blood flow. Brain Res 535: 313-317

Deschaumes-Molinaro C, Dittmar A, Sicard G, VernetMaury E (1992) Results from six autonomic nervous system confirms autonomic response specificity hypothesis. Hom in Health and Disease 33: 225-239

Deschaumes-Molinaro C, Dittmar A, Vernet-Maury E (1991) Relationship between mental imagery and sporting performance. Behav Brain Res 45: 29-36

Drummond P, White K, Ashton R (1978) Imagery vividness affects habituation rate. Psychophysiol 15: 193-195

Gandevia SC, Hall LA, McCloskey DI, Potter EK (1983) Anticipation sensation at the terminal joint of the middle finger. J Physiol 355: 507-517

Hale BD (1982) The effects of internal and external imagery on muscular and ocular concomitants. J Sports Psychol 4: 379-387

Ingvar DH, Philipson L (1977) Distribution of cerebral blood flow in the dominant hemisphere motor ideation and motor performance. Annal Neurol 2: 230-237

Lacey PJ, Lacey BC (1970) Some autonomic-central nervous system inter-relations. In Black $\mathrm{P}$ ed. Physiological correlates of emotion, Academic Press, New York, 205-227

Lang PJ, Öhman A, Simons RF (1978) The psychophysiology of anticipation. In Requin $\mathrm{J}$ ed. Attention and performance VII, Lawrence Erlbaum Associates, Hillsdale, 469-485

Mahoney MJ, Avener M (1977) Psychology of the elite athlete: An exploratory study. Cogn Ther Res 1: 135-
141

Oishi K, Kimura M, Yasukawa M, Maeshima T (1992) Changes of physiological parameters during mental rehearsal of speed skating. Jpn J Physical Education 36: 303-312 (in Japanese)

Oishi K, Kimura M, Yasukawa M, Yoneda T, Maeshima T (1994) Amplitude reduction of H-reflex during mental movement simulation in elite athletes. Behav Brain Res 62: 55-61

Pauli P, Lutzenberger W, Rau H, Birbaumer N, Rickard TC, Yaroush RA, Bourne Jr LE (1994) Brain potentials during mental arithmetic: effects of extensive practice and problem difficulty. Cogn Brain Res 2: 21-29

Roland PE, Larsen B, Lassen NA, Skinhoj E (1980) Supplementary motor area and other cortical area in organization of voluntary movements in man. J Neurophysiol 43: 118-136

Ryding E, Decety J, Sjoholm H, Stenberg G, Ingvar DH (1993) Motor imagery activates the cerebellum regionally. A SPECT rCBF study with 99mTc-HMPAO. Cogn Brain Res 1: 94-99

Tobin MJ, Perez W, Guenther SM, D'Alonzo G, Dantzker DR (1986) Breathing pattern and metabolic behavior during anticipation of exercise. J Appl Physiol 60: 1306-1312.

Wallin BG, Fagius J (1986) The sympathetic nervous system in man: aspects derived from microelectrode recordings. Trends in Neurosci 9: 63-67

Wang Y, Mogan W (1992) The effect of imagery perspectives on the psychophysiological responses to imagined exercise. Behav Brain Res 52: 167-174

Wuyam B, Moosavi SH, Decety J, Adams L, Lansing RW, Guz A (1995) Imagination of dynamic exercise produced ventilatory responses which were more apparent in competitive sportsmen. J Physiol 482: 713724

Yasukawa M, Oishi K, Kimura M, Maeshima T (1993) Accuracy of time estimation during mental rehearsal. Jpn J Physical Education 37: 385-391 (in Japanese)

Yue G, Cole KJ (1993) Strength increases from the motor program: comparison of training with maximal voluntary and imagined muscle contractions. J Neurophysiol 67: 1114-1123

Received: March 8, 2000

Accepted: September 21, 2000

Correspondence to: Kazuo Oishi, Health and Sports Sciences Institute, Senshu University, 2-1-1, Higashimita, Tama, Kanagawa 214-8580, Japan e-mail: QWK02752@nifty.ne.jp 